INPLASY

PROTOCOL

To cite: Wang et al.

Association between the

CYP17A1 - 34T/C

polymorphism and polycystic

ovary syndrome: A meta-

analysis. Inplasy protocol

2020110081. doi:

10.37766/inplasy2020.11.0081

Received: 19 November 2020

Published: 19 November 2020

Corresponding author:

Zhi Cheng

zcheng@cqmu.edu.cn

Author Affiliation:

Chongqing Medical University

Support: No.2016CSTC-

jbky-01711.

Review Stage at time of this submission: Data analysis.

Conflicts of interest:

The authors have declared that no competing interests exist.

\section{Association between the CYP17A1 - 34T/C polymorphism and polycystic ovary syndrome: A meta-analysis}

Wang, Y1; Duan, Z2; Wang, Y3; He, X4; Cheng, Z5.
Review question / Objective: To examine the hypothesis that CYP17A1 -34T/C polymorphism is associated with and polycystic ovary syndrome.

Condition being studied: Polycystic ovary syndrome.

Information sources: We searched PubMed, Web of Science and Chinese Nation Knowledge Infrastructure (CNKI) databases to retrieve eligible studies.

INPLASY registration number: This protocol was registered with the International Platform of Registered Systematic Review and Meta-Analysis Protocols (INPLASY) on 19 November 2020 and was last updated on 19 November 2020 (registration number INPLASY2020110081).

\section{INTRODUCTION}

Review question / Objective: To examine the hypothesis that CYP17A1 -34T/C polymorphism is associated with and polycystic ovary syndrome.

Rationale: To date, many studies have been performed to evaluate the association between the CYP17A1-34T/C polymorphism and polycystic ovary syndrome (PCOS). However, due to differences in sample size and ethnicity, the results are inconclusive. Therefor, a metaanalysis based on all currently available published data was performed to characterize more precisely the potential 
association between the CYP17A1 -34T/C polymorphism and PCOS.

Condition being studied: Polycystic ovary syndrome.

\section{METHODS}

Search strategy: A systematic published literature search in the PubMed, Web of Science and Chinese Nation Knowledge Infrastructure (CNKI) databases was conducted. The following key words were used: "CYP17 or CYP17A1", "polymorphism", "PCOS or polycystic ovary syndrome".

Participant or population: Studies that evaluating the association between the CYP17A1 -34T/C polymorphism and PCOS in cases and controls. Studies were excluded if genotype distribution in the control group deviated from the HardyWeinberg equilibrium, or they did not have sufficient genotype data were presented to estimate the odds ratios and $95 \%$ confidence intervals, or there was mixed sexes in controls.

Intervention: CYP17A1 - 34T/C polymorphism.

\section{Comparator: Healthy women.}

Study designs to be included: Casecontrol.

Eligibility criteria: Studies that evaluating the association between the CYP17A1 34T/C polymorphism and PCOS in cases and controls. Studies were excluded if genotype distribution in the control group deviated from the Hardy-Weinberg equilibrium, or they did not have sufficient genotype data were presented to estimate the odds ratios and $95 \%$ confidence intervals, or there was mixed sexes in controls.

Information sources: We searched PubMed, Web of Science and Chinese Nation Knowledge Infrastructure (CNKI) databases to retrieve eligible studies.
Main outcome(s): We found that the CYP17A1 -34T/C polymorphism is associated with PCOS development in South Asian and West Asian populations, but not in Caucasian and East Asian populations.

Data management: Two investigators independently extracted the usable data from all included studies. Any divergences of opinion were resolved by discussion between the two investigators. The following data were collected from each study: first author's last name, year of publication, country of origin, ethnicity (Caucasian, Asian), genotyping methods, and the number of cases and controls for each genotype.

Quality assessment / Risk of bias analysis: The methodological quality of each eligible study was independently assessed by two investigators according to the NewcastleOttawa Scale (NOS). Each study was evaluated and scored based on the selection, comparability, and exposure of the study group. NOS scores ranged from 0 to 9 and studies with a score $\geq 7$ were considered high quality.

Strategy of data synthesis: We analysed the association between the CYP17A1 -34T/C polymorphism and PCOS under allele model, homozygous gene model, heterozygous gene model, dominant gene model, and recessive gene model, respectively. Heterogeneity across the studies was tested by the I-squared statistic (12). The odds ratio (OR) and corresponding $95 \%$ confidence interval $(95 \% \mathrm{Cl})$ were calculated to estimate the strength of the association between the CYP17A1 -34T/C polymorphism and PCOS. Begg's and Egger's tests and funnel plots were used to examine the publication bias.

Subgroup analysis: Stratified analyses were performed based on ethnicity: Caucasian, East Asian South Asian and West Asian. Begg's and Egger's tests and funnel plots were also conduct to examine the publication bias in each subgroup. 
Sensibility analysis: Sensitivity analysis, which involves sequential exclusion of one study at a time and recalculation of the pooled ORs, was used to examine the stability and reliability of the overall results.

Language: English.

Country(ies) involved: China.

Keywords: Meta-analysis, CYP17A1, polymorphism, polycystic ovary syndrome.

Contributions of each author:

Author 1 - Yuanyuan Wang - Formal analysis; Conceptualization; Investigation; Methodology.

Author 2 - Zhaoning Duan - Formal analysis; Conceptualization; Investigation; Methodology.

Author 3 - Yunpeng Wang - Investigation.

Author 4 - Xiwen He - Investigation.

Author 5 - Zhi Cheng - Conceptualization;

Funding acquisition; Methodology;

Supervision. 